ARTICLE

DOI: $10.1038 / s 41467-018-05363-6$

\title{
Long range electronic phase separation in $\mathrm{CaFe}_{3} \mathrm{O}_{5}$
}

\author{
Ka. H. Hong (1) ${ }^{1}$, Angel M. Arevalo-Lopez ${ }^{2}$, James Cumby (1) ${ }^{1},{\text { Clemens } \text { Ritter }^{3} \& \text { J. Paul Attfield (1) }}^{1}$
}

Incomplete transformations from ferromagnetic to charge ordered states in manganite perovskites lead to phase-separated microstructures showing colossal magnetoresistances. However, it is unclear whether electronic matter can show spontaneous separation into multiple phases distinct from the high temperature state. Here we show that paramagnetic $\mathrm{CaFe}_{3} \mathrm{O}_{5}$ undergoes separation into two phases with different electronic and spin orders below their joint magnetic transition at $302 \mathrm{~K}$. One phase is charge, orbital and trimeron ordered similar to the ground state of magnetite, $\mathrm{Fe}_{3} \mathrm{O}_{4}$, while the other has $\mathrm{Fe}^{2+} / \mathrm{Fe}^{3+}$ charge averaging. Lattice symmetry is unchanged but differing strains from the electronic orders probably drive the phase separation. Complex low symmetry materials like $\mathrm{CaFe}_{3} \mathrm{O}_{5}$ where charge can be redistributed between distinct cation sites offer possibilities for the generation and control of electronic phase separated nanostructures.

\footnotetext{
${ }^{1}$ Centre for Science at Extreme Conditions and School of Chemistry, University of Edinburgh, Mayfield Road, Edinburgh EH9 3JZ, UK. ${ }^{2}$ Univ. Lille, CNRS, Centrale Lille, ENSCL, Univ. Artois, UMR 8181 - UCCS - Unité de Catalyse et Chimie du Solide, Lille F-59000, France. ${ }^{3}$ Institut Laue-Langevin, 71 avenue des Martyrs, Grenoble 38000, France. Correspondence and requests for materials should be addressed to J.P.A. (email: j.p.attfield@ed.ac.uk)
} 
M

anganites such as $\mathrm{La}_{0.7} \mathrm{Ca}_{0.3} \mathrm{MnO}_{3}$ and magnetite, $\mathrm{Fe}_{3} \mathrm{O}_{4}$, share similar physics as both have a spinpolarised conducting state near ambient temperature due to double exchange between ferromagnetically aligned $\mathrm{Mn}^{3+}$ and $\mathrm{Mn}^{4+}$ or $\mathrm{Fe}^{2+}$ and $\mathrm{Fe}^{3+}$ spins, respectively. Both undergo charge ordering on cooling, which for magnetite is accompanied by a lattice distortion below the much-studied Verwey transition at $125 \mathrm{~K}^{1}$. This was recently found to result from a complex ordering of $\mathrm{Fe}^{2+} / \mathrm{Fe}^{3+}$ charge states, $\mathrm{Fe}^{2+}$ orbitals, and three-Fe trimeron groups ${ }^{2}$. However, long range phase segregation is observed below charge ordering transitions in many manganites $^{3-5}$ and related perovskites but has not been found in magnetite or related ferrite spinels.

Here, following recent studies of spin and charge ordering in non-spinel $M \mathrm{Fe}_{n-1} \mathrm{O}_{n+1}(n \geq 4)$ ferrites with $M=\mathrm{Fe}^{6,7}, \mathrm{Mn}^{8,9}$ and $\mathrm{Ca}^{10,11}$, we investigate $n=4 \mathrm{CaFe}_{3} \mathrm{O}_{5}$ and discover long range electronic phase separation between a magnetite-like charge ordered phase and a charge averaged phase.

\section{Results}

Preparation and characterisation of $\mathrm{CaFe}_{3} \mathrm{O}_{5} \cdot \mathrm{CaFe}_{3} \mathrm{O}_{5}$ was synthesised and characterised as described in Methods. The orthorhombic crystal structure consists of $\mathrm{FeO}_{6}$ octahedra sharing edges to form infinite chains parallel to the $a$-axis and ribbons of three octahedra parallel to the $b c$-plane as shown in Fig. 1a. Magnetisation measurements (Fig. 1b) reveal a sharp magnetic ordering transition at $T_{\mathrm{M}}=302 \mathrm{~K}$ and a small net magnetisation of $0.05 \mu_{\mathrm{B}}$ per $\mathrm{CaFe}_{3} \mathrm{O}_{5}$ unit with a moderate coercivity of $0.3 \mathrm{~T}$ at $2 \mathrm{~K}$. Ceramic pellets of $\mathrm{CaFe}_{3} \mathrm{O}_{5}$ are semiconducting with a small magnetoresistance of $-1 \%$ at $200 \mathrm{~K}$ (Fig. 1c), likely reflecting the small net magnetisation of the sample.

Evidence for electronic phase separation in $\mathrm{CaFe}_{3} \mathrm{O}_{5}$. A single high temperature (HT) crystalline phase of $\mathrm{CaFe}_{3} \mathrm{O}_{5}$ is observed above $T_{\mathrm{M}}=302 \mathrm{~K}$, but both powder synchrotron X-ray (Fig. 2a, b) and neutron diffraction data (Fig. 2c, d) reveal long range phase separation as diffraction peaks broaden or split into two components below the magnetic ordering transition as shown in Fig. 2a, c. The shift of the X-ray (002) peak to lower $2 \theta$ at $300 \mathrm{~K}$ evidences a small bulk lattice distortion due to spin ordering before separation into two phases at lower temperatures. Both low temperature phases have the same $\mathrm{Cmcm}$ space group symmetry as the HT parent. Crystallographic results are shown in Supplementary Tables 1-5. Magnetic neutron diffraction peaks observed below $T_{\mathrm{M}}$ (Supplementary Fig. 1) show that one phase has magnetic propagation vector $\left(\begin{array}{lll}1 / 2 & 0 & 0\end{array}\right)$ while the other has $\left(\begin{array}{lll}0 & 0 & 0\end{array}\right)$ (Fig. 2c), and a good fit to the data (Fig. 2d) was obtained using the magnetic structure models shown in Fig. 3. No additional broadening of magnetic peaks was observed showing that both magnetic orders have correlation lengths of at least $200 \mathrm{~nm}$. Both phases have collinear antiferromagnetic orders with moments parallel to the $c$-axis, but an important difference is that the $\left(\begin{array}{lll}1 / 2 & 0 & 0\end{array}\right)$ phase has ferromagnetic (FM) alignment of spins within the three-atom ribbons parallel to the $b c$-plane, while the $\left(\begin{array}{lll}0 & 0 & 0\end{array}\right)$ has FM spin chains parallel to the $a$-axis. Although both orders are antiferromagnetic, the observation of a small net magnetisation below $T_{\mathrm{M}}$ suggests that one or both of the spin structures are canted. The fractions of the two phases are found to be different in the synchrotron and neutron diffraction experiments (Fig. 2a), consistent with a strain-driven phase separation being dependent on the thermal history of the sample.

Thermal evolution of lattice parameters from neutron and Xray fits are, respectively, shown in Fig. 4a and Supplementary Fig. 2, and variations of internal structural quantities from both studies are shown in Fig. 4b-d to emphasise the reproducibility of
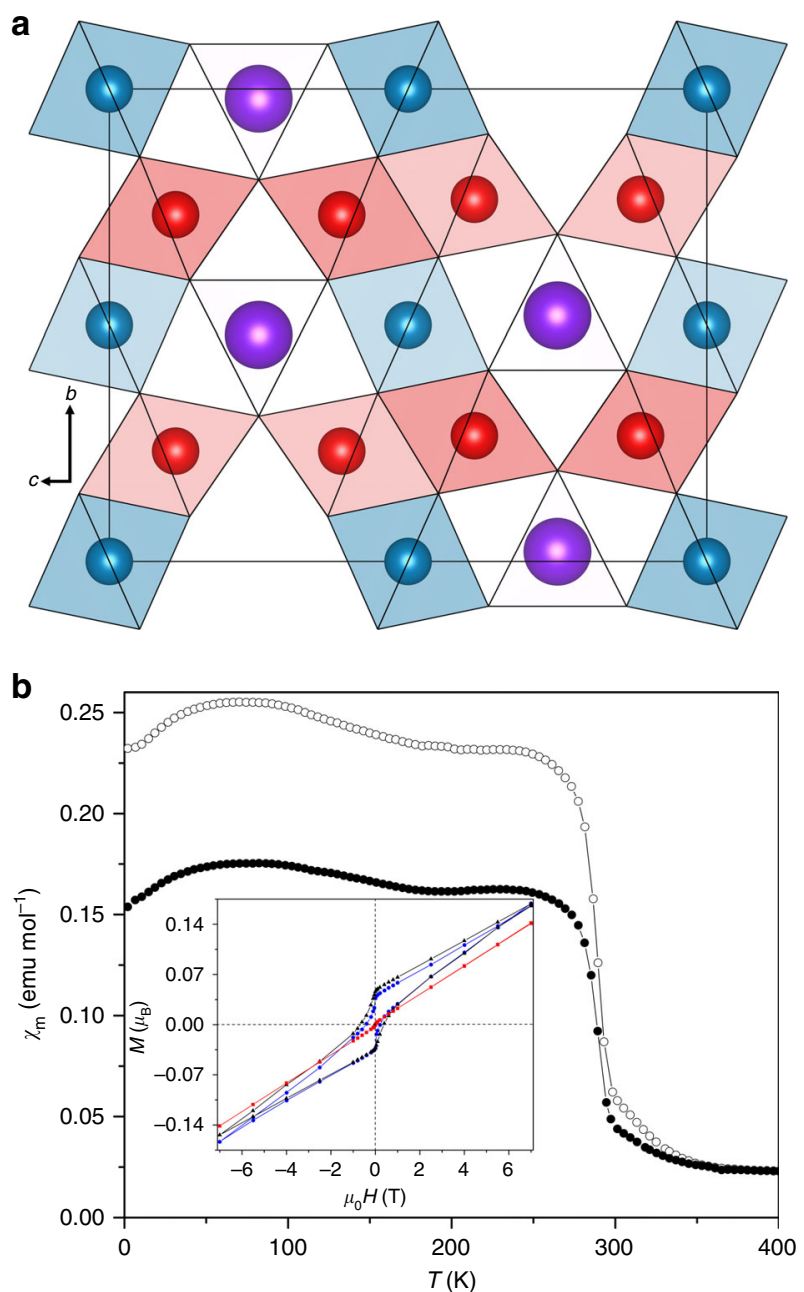

C

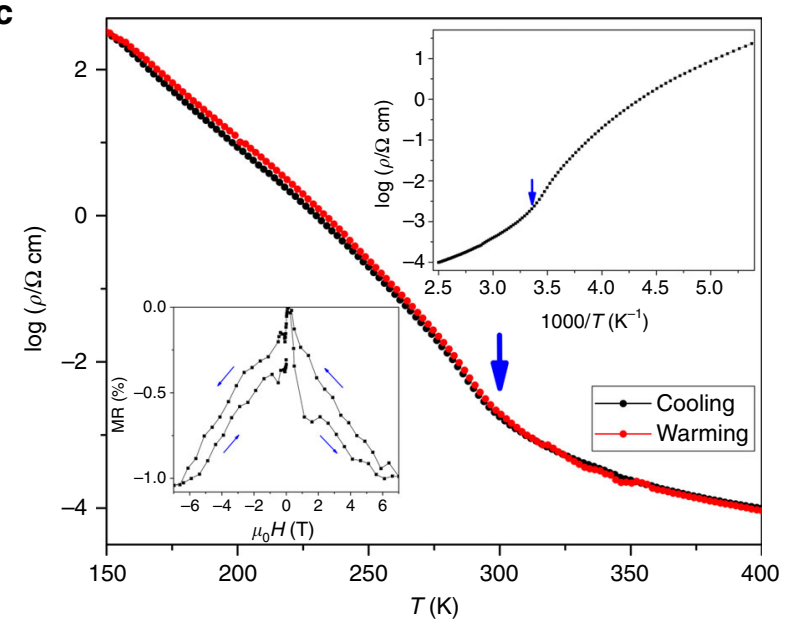

Fig. 1 Crystal structure and magnetisation studies of $\mathrm{CaFe}_{3} \mathrm{O}_{5}$. a Polyhedral projection of the $\mathrm{CaFe}_{3} \mathrm{O}_{5}$ structure showing $\mathrm{FeO}_{6}$ octahedra in red/blue for symmetry inequivalent $\mathrm{Fe} 1 / \mathrm{Fe} 2$ sites, and $\mathrm{Ca}$ within trigonal prismatic tunnels in purple. b ZFC (closed symbols) and FC (open symbols) magnetic susceptibilities for $\mathrm{CaFe}_{3} \mathrm{O}_{5}$, with insert showing magnetisation-field measurements at 2, 230 and $300 \mathrm{~K}$ (blue, black and red points respectively). c $\log _{10}$ plot of the electrical resistivity of a sintered pellet of $\mathrm{CaFe}_{3} \mathrm{O}_{5}$ against temperature on cooling and warming, with the discontinuity at $302 \mathrm{~K}$ marked. Inset in the top right shows the plot against reciprocal temperature with a change in activation energy from $0.61 \mathrm{eV}$ below $T_{M}$ to $0.26 \mathrm{eV}$ above the transition. Inset in lower left displays the magnetoresistance MR at $200 \mathrm{~K}$ 
discovered structural differences between the two phases. Two inequivalent iron sites $\mathrm{Fe} 1$ and $\mathrm{Fe} 2$ are present in a 2:1 ratio in $\mathrm{CaFe}_{3} \mathrm{O}_{5}$, and in the HT phase they have respective Bond Valence Sums (BVSs) of 2.43 and 2.22, equivalent to formal charges of +2.75 and +2.50 when renormalized to the average of +2.67 . Differing charge redistributions occur below the $302 \mathrm{~K}$ magnetic and phase segregation transition (Fig. $4 \mathrm{~b}$ ). In the phase with magnetic propagation vector $(1 / 200)$, the $\mathrm{Fe} 1$ and $\mathrm{Fe} 2$ BVSs diverge to very different values of $2.67(6)$ and $2.03(4)$ at $4 \mathrm{~K}$, consistent with charge order $(\mathrm{CO})$ of $\mathrm{Fe}^{3+}$ and $\mathrm{Fe}^{2+}$ respectively. The coexisting phase with $\left(\begin{array}{lll}0 & 0 & 0\end{array}\right)$ magnetic order shows the opposite behaviour as the $\mathrm{Fe} 1$ and Fe2 BVSs converge to similar values of 2.44(8) and 2.29(7) at $4 \mathrm{~K}$ showing that the electronic states at the two sites are not significantly different, hence this phase is charge averaged (CA).

Degenerate $3 \mathrm{~d}^{6} \mathrm{Fe}^{2+}$ states are subject to Jahn-Teller distortion which leads to tetragonal octahedral compression in magnetite ${ }^{2}$, corresponding to negative values of the $Q_{\mathrm{TT}}$ distortion parameter. The $\mathrm{Fe} 2$ site in $\mathrm{CaFe}_{3} \mathrm{O}_{5}$ has a large negative $Q_{\mathrm{TT}}$ value due to intrinsic distortions within the HT structure (Fig. 4c), but below $T_{\mathrm{M}}=302 \mathrm{~K} Q_{\mathrm{TT}}$ becomes more negative for the CO phase, consistent with localisation of $\mathrm{Fe}^{2+}$ states, while the magnitude of $Q_{\mathrm{TT}}$ decreases in keeping with the observed increase in Fe2 site valence for the CA phase. Jahn-Teller distortion at the Fe2 site in the CO phase leads to order of the $t_{2 g}$ orbital with minority spin electron density directed towards the two neighbouring Fe1 sites. Hence this localised orbital has the correct orientation to form a trimeron, a linear unit of three $\mathrm{Fe}$ ions formed by delocalisation of the minority spin electron in the ordered orbital of the central $\mathrm{Fe}^{2+}$ ion ${ }^{2}$, as shown in Fig. 3c. Trimeron formation requires FM alignment of the three magnetic moments, $\mathrm{Fe}^{3+}-\mathrm{Fe}^{2+}-\mathrm{Fe}^{3+} \mathrm{CO}$, orbital order at the central $\mathrm{Fe}^{2+}$ in the plane of the three atoms, and shortening of the $\mathrm{Fe}-\mathrm{Fe}$ distances within the trimeron ${ }^{2}$. All these conditions are observed in the $\mathrm{Fe} 1-\mathrm{Fe} 2-\mathrm{Fe} 1$ ribbons in the $\mathrm{CO}$ phase with shortening of $\mathrm{Fe} 1-\mathrm{Fe} 2$ distances below $T_{\mathrm{M}}$ observed in Fig. $4 \mathrm{~d}$, but none are fulfilled in the CA structure, so the observed spin orders and structural distortions in $\mathrm{CaFe}_{3} \mathrm{O}_{5}$ demonstrate that trimeron order is observed only in the $\mathrm{CO}$ phase. However, a slight shortening of Fe-Fe distances in the FM chains parallel to the $a$-axis is observed in the CA phase (Fig. 4d), consistent with a weak bonding effect from extended band states of the minority spin electrons. Fe1-Fe1 and $\mathrm{Fe} 2-\mathrm{Fe} 2$ distances are constrained to be equal (to $a / 2$ ) and this is the likely driver for the observed averaging of charge states between the two sites in the CA phase so that their minority spin populations become more equal.
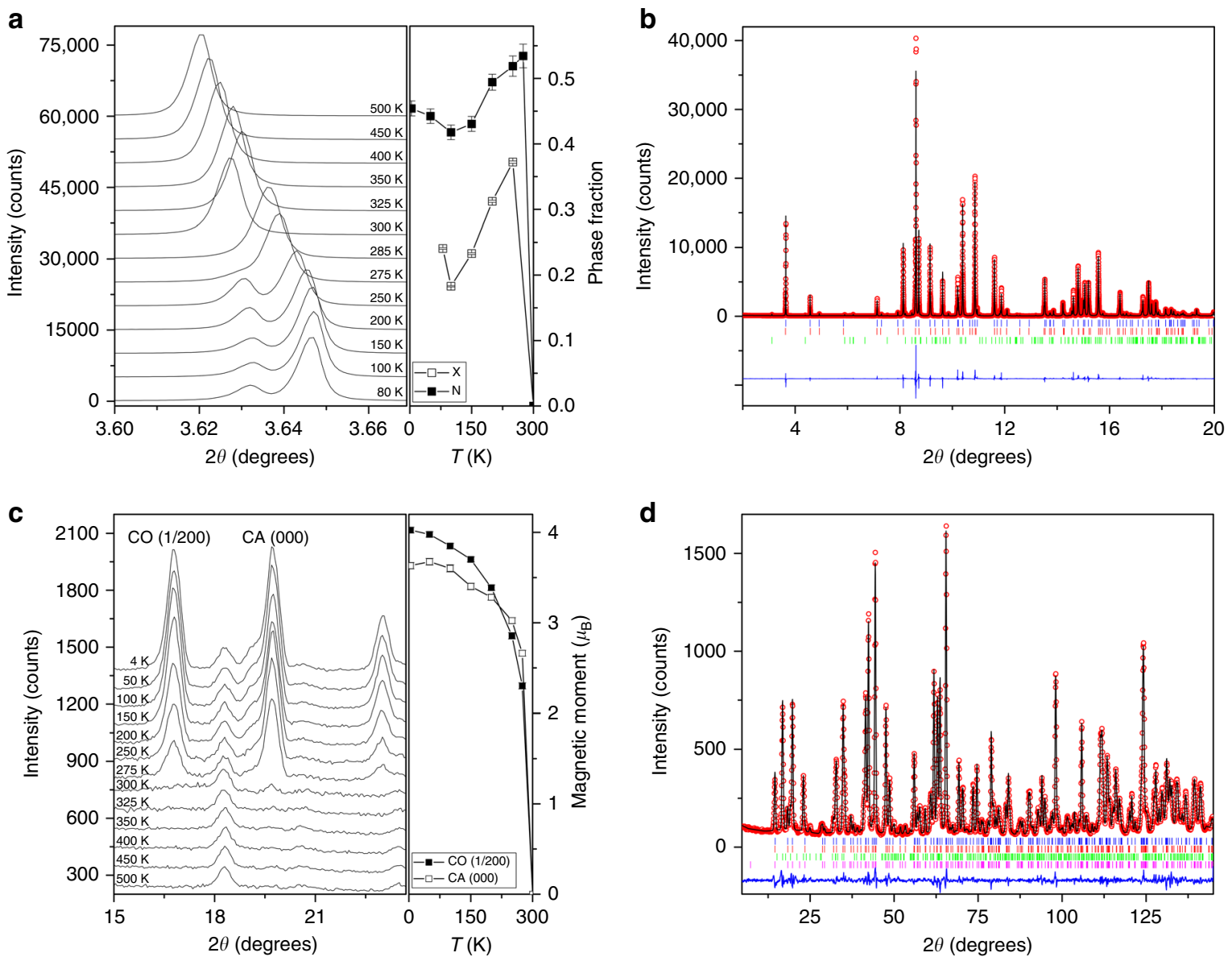

Fig. 2 Electronic phase separation of $\mathrm{CaFe}_{3} \mathrm{O}_{5}$. a Evolution of the X-ray (002) peak which shifts to lower $2 \theta$ when cooled to $300 \mathrm{~K}$, just below the $T_{\mathrm{M}}=302 \mathrm{~K}$ transition, with separation into two components at lower temperatures. The phase fractions of the charge averaged (CA) phase, obtained from synchrotron $(\mathrm{X})$ and neutron $(\mathrm{N})$ diffraction data are shown in the right-hand panel. Error bars are the estimated standard deviations calculated during profile fitting. $\mathbf{b}$ Rietveld fit to synchrotron powder diffraction profiles for $\mathrm{CaFe}_{3} \mathrm{O}_{5}$ at $80 \mathrm{~K}$ ( $\mathrm{R}$-factors $R_{\mathrm{p}}=9.35 \%, R_{\mathrm{wp}}=11.0 \%$ ), with blue and red tick marks indicating the two low temperature phases and green marks $2.3 \%$ of $\mathrm{Ca}_{2} \mathrm{Fe}_{2} \mathrm{O}_{5}$ impurity. c The appearance of the magnetic reflections with propagation vectors of $(000)$ for the $\mathrm{CO}$ phase and $(1 / 200)$ for the charge averaged (CA) phase below $302 \mathrm{~K}$ and the temperature evolution of their magnetic moments. d The Rietveld fit to neutron powder diffraction profiles for $\mathrm{CaFe}_{3} \mathrm{O}_{5}$ at $4 \mathrm{~K}$ (R-factors $R_{\mathrm{p}}=6.14 \%, R_{\mathrm{wp}}=7.28 \%$ ), with structural phases indicated with blue and red tick marks. The green and pink tick marks represent the magnetic phases with propagation vectors of $(1 / 200)$ and $(000)$ 
a

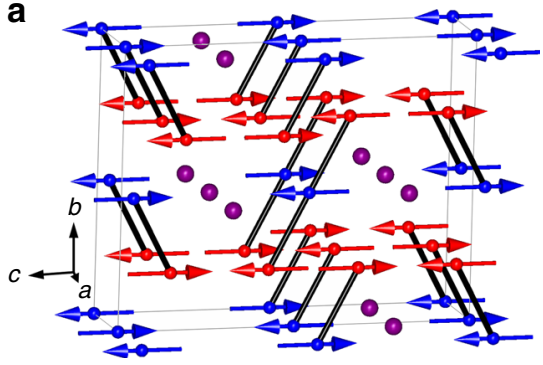

b

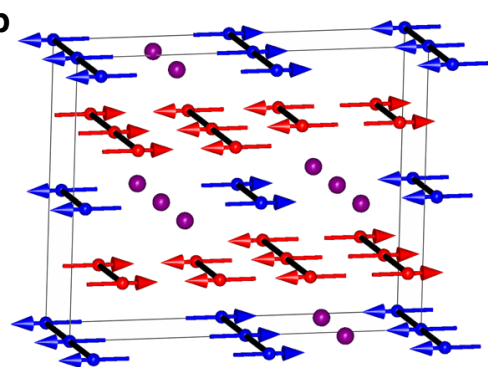

C

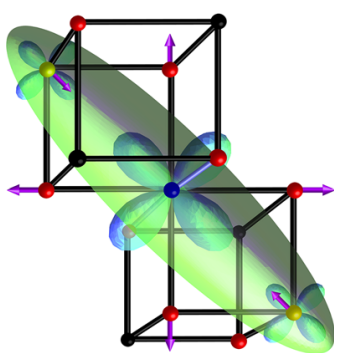

Fig. 3 Magnetic structures for the two phases of $\mathrm{CaFe}_{3} \mathrm{O}_{5}$. $\mathrm{Fe} 1 / \mathrm{Fe} 2$ spins are shown in red/blue and $\mathrm{Ca}$ ions in purple. a Magnetic structure of the charge ordered $(\mathrm{CO})$ phase $\mathrm{CaFe}_{3} \mathrm{O}_{5}$ with propagation vector $(1 / 2 \mathrm{O} 0)$, with lines showing ferromagnetic order within trimerons. $\mathbf{b}$ Magnetic structure of the charge averaged ( $C A$ ) phase, with $(000)$ propagation and lines showing the ferromagnetic chain parallel to the $x$ axis. $\mathbf{c} A$ trimeron unit as found in the $\mathrm{CO}$ structure (a) with bonding electron density represented as an ellipsoid. The size of the $t_{2 g}$ orbitals approximates the atomic populations. The atomic displacement arrows indicate the elongation of the four Fe-O bonds perpendicular to the Jahn-Teller contracted axis and the shortening of the cation-cation distances due to weak Fe-Fe bonding interactions
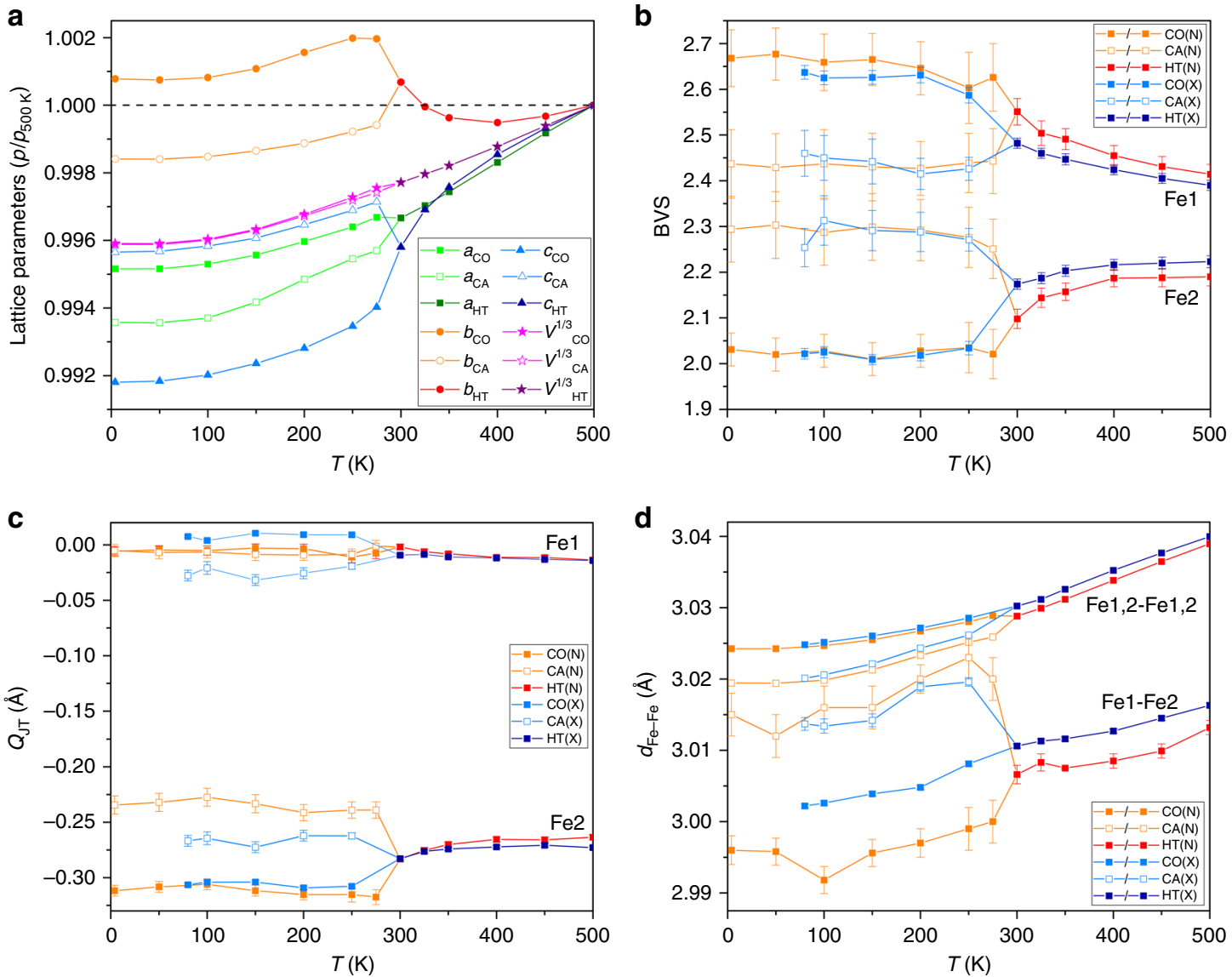

Fig. 4 Crystal structure parameters of $\mathrm{CaFe}_{3} \mathrm{O}_{5}$. a Changes in the lattice parameters relative to $500 \mathrm{~K}$ values obtained from PND $\left(a_{500 \mathrm{~K}}=3.03896(1)\right.$, $b_{500}$ $\mathrm{K}=10.01355(5)$ and $\left.c_{500 \mathrm{~K}}=12.67039(7) \AA\right)$. Temperature dependence of the $\mathbf{b} \mathrm{BVS}$ and $\mathbf{c}$ the amplitude of Jahn-Teller distortion ( $\left.\mathrm{Q}_{\mathrm{JT}}\right)$ of the FeO 6 octahedra for the two sites in the high temperature $(\mathrm{HT})$, charge ordered (CO) and charge averaged (CA) phases, obtained from neutron (N) and synchrotron $\mathrm{X}$-ray $(\mathrm{X})$ diffraction data. $\mathbf{d}$ The evolution of the Fe1-Fe2 bonding distance with temperature, with the lattice parameter (Fe1,2-Fe1,2) as reference. Error bars are the estimated standard deviations calculated during profile fitting

The electronic orders in the two phases of $\mathrm{CaFe}_{3} \mathrm{O}_{5}$ do not change the structural symmetry, but they lead to different cell distortions (Fig. 4a) as trimeron formation shortens the $c$-axis parameter in the $\mathrm{CO}$ phase, with $a$ and $b$ expanding to compensate, while the $a$-axis shortening in the CA phase leads to expansion of $c$. Although the overall cell volumes for the two components remain identical within error at low temperatures, the differing spontaneous strains from these lattice distortions are the likely driver for the long range separation as proposed for perovskite manganites ${ }^{12-14}$. Short range phase and strain fluctuations in the HT phase are evidenced indirectly below $350 \mathrm{~K}$ through divergence of zero field- and field- cooled susceptibilities in Fig. 1b, and changes in the slopes of $b$ and $c$ lattice parameters in Fig. 4a.

Charge ordering in one of the two low temperature phases is clearly a key driver for the long range electronic phase separation in $\mathrm{CaFe}_{3} \mathrm{O}_{5}$ and perovskite type oxides ${ }^{15}$ although in $\mathrm{CaFe}_{3} \mathrm{O}_{5}$ this leads to the formation of orbital molecules ${ }^{16}$, more complex 
electronic objects than simple localised-charge ions. However, phase separation in $\mathrm{CaFe}_{3} \mathrm{O}_{5}$ occurs without a change of lattice symmetry in either component, demonstrating that strain variations within a given lattice are sufficient to drive long range segregation. Band structure calculations (shown in Supplementary Fig. 4 and Supplementary Table 6) confirm that these distortions are sufficient to stabilise $\mathrm{CO}$ in one structure but not the other for a realistic value of the Hubbard U-parameter. Phase separation in manganites usually results from an incomplete phase transition where a metallic HT FM phase is partially transformed to a charge ordered insulator, and the high and low temperature phases coexist to base temperature. A notable example is $\mathrm{Nd}_{0.5} \mathrm{Sr}_{0.5} \mathrm{MnO}_{3}$ which on cooling was observed to order as a single FM phase at $250 \mathrm{~K}$, then to phase separate into a mixture of FM and A-type antiferromagnetic (AFM-A) phases below $220 \mathrm{~K}$, and finally to undergo a further phase separation into a mixture of FM, AFM-A and charge ordered AFM-CE phases below $150 \mathrm{~K}$, with all three phases extant down to the lowest measured temperature of $15 \mathrm{~K}^{17} \cdot \mathrm{CaFe}_{3} \mathrm{O}_{5}$ undergoes a genuine electronic phase separation in the sense that both of the two low temperature CA and $\mathrm{CO}$ phases are electronically and magnetically distinct from the HT paramagnetic state, and so is analogous to e.g. the separation of a fluid into a liquid and gas below a critical transition. Both low temperature $\mathrm{CaFe}_{3} \mathrm{O}_{5}$ phases are magnetically ordered and it is remarkable that they appear to share a common magnetic ordering temperature as shown in Fig. $2 \mathrm{c}$ although the spin-spin exchange interactions within the two magnetic structures will not be identical.

Another important difference is that phase separation in manganites is associated with substantial intrinsic disorder due to cation mixing, and a recent study has demonstrated that chemical ordering of cations in $\left(\mathrm{La}_{1-\gamma} \mathrm{Pr}_{y}\right)_{1-\mathrm{x}} \mathrm{Ca}_{x} \mathrm{MnO}_{3}$ suppresses long range phase separation ${ }^{18}$. $\mathrm{CaFe}_{3} \mathrm{O}_{5}$ is in principle a stoichiometric material although small strain variations due to the $4 \%$ substitution of $\mathrm{Fe}$ for $\mathrm{Ca}$ observed in our polycrystalline sample may tip the local balance between the energies of the two ground states leading to phase coexistence. The resulting disorder in magnetic interactions could also be important in stabilising the phase coexistence in a Griffiths-type model ${ }^{19}$. All Mn sites are electronically equivalent in the $\mathrm{HT}$ aristotype manganite perovskite structure but $\mathrm{CaFe}_{3} \mathrm{O}_{5}$ has a further electronic degree of freedom as charge can be redistributed between structurally inequivalent $\mathrm{Fe} 1$ and $\mathrm{Fe} 2$ sites, leading to the observed extremes of charge ordering $(\mathrm{CO})$ in one phase and charge averaging (CA) in the other. This charge redistribution mechanism is akin to electronic separation models originally proposed for manganites ${ }^{20,21}$, although couplings of both the charge redistribution degree of freedom and trimeron formation associated with charge and orbital ordering to the lattice appear to be important factors that drive phase segregation in $\mathrm{CaFe}_{3} \mathrm{O}_{5}$.

\section{Discussion}

In conclusion, the present study demonstrates that phase separation of a single HT paramagnetic state into two distinct low temperature phases with different long range spin and electronic orders occurs in $\mathrm{CaFe}_{3} \mathrm{O}_{5}$ when cooled below $302 \mathrm{~K}$. Although both phases are antiferromagnetic overall, the formation of different FM units within them is coupled to the electronic orders; one phase has full $\mathrm{Fe}^{2+} / \mathrm{Fe}^{3+} \mathrm{CO}$ associated with trimeron formation, but CA stabilises FM chains in the other phase. Weak Fe-Fe bonding driven by the FM orders introduces different lattice strains into the two phases although no change of structural symmetry occurs, and strain variations within the polycrystalline sample tip the local balance between the energies of the two phases leading to phase coexistence. $\mathrm{CaFe}_{3} \mathrm{O}_{5}$ thus links the trimeron-ordering of magnetite to the microstructural physics of perovskite manganites. $\mathrm{CaFe}_{3} \mathrm{O}_{5}$ also demonstrates new possibilities for more complex 'electronically soft matter'22 than in perovskites where orbital molecule formation and redistribution of charge between distinct cation sites offer additional degrees of freedom for generation and potential control of electronic phase-segregated nanostructures.

\section{Methods}

Sample preparation and characterisation. Polycrystalline $\mathrm{CaFe}_{3} \mathrm{O}_{5}$ was prepared from stoichiometric quantities of $\mathrm{CaFe}_{2} \mathrm{O}_{4}, \mathrm{Fe}_{2} \mathrm{O}_{3}$, and $\mathrm{Fe}$ powders pressed into pellets, sealed in evacuated quartz tubes, and heated at $1100^{\circ} \mathrm{C}$ for $12 \mathrm{~h}$. (The $\mathrm{CaFe}_{2} \mathrm{O}_{4}$ was synthesised at ambient pressure using the ceramic technique outlined by Wan et $\mathrm{al}^{23}$. where $\mathrm{CaCO}_{3}$ and $\mathrm{Fe}_{2} \mathrm{O}_{3}$ powders were ground together in 1:1 ratio, pressed into pellets, heated at $850^{\circ} \mathrm{C}$ for $4 \mathrm{~h}$, reground and repelleted, and finally reheated at $1100^{\circ} \mathrm{C}$ for $12 \mathrm{~h}$.) Thermogravimetric analysis heating the sample in air at $10^{\circ} \mathrm{C} \mathrm{min}-1$ to $900^{\circ} \mathrm{C}$, as shown in Supplementary Fig. 3, gave a mass increase of $2.789 \%$, in agreement with the calculated value of $2.781 \%$ for oxidation of $\mathrm{CaFe}_{3} \mathrm{O}_{5}$. Powder X-ray diffraction confirmed that $\mathrm{CaFe}_{3} \mathrm{O}_{5}$ adopts an orthorhombic $\mathrm{Sr}_{2} \mathrm{Tl}_{2} \mathrm{O}_{5}$ type structure with space group $\mathrm{Cmcm}$ as reported previously ${ }^{10}$.

Magnetic and electrical measurements. Magnetic measurements were carried out with a Quantum Design MPMS XL SQUID magnetometer. Magnetic susceptibility was recorded in zero field cooled (ZFC) and field cooled (FC) conditions between 2 and $400 \mathrm{~K}$ with an applied magnetic field of 1000 Oe. Hysteresis loops were also measured at 2, 230 and $300 \mathrm{~K}$. Electrical resistivity measurements were carried out with a Quantum Design PPMS, between 180 and $400 \mathrm{~K}$. Magnetoresistance hysteresis loops were also measured at $200 \mathrm{~K}$.

Powder synchrotron X-ray and neutron diffraction studies. High resolution powder X-ray diffraction data were collected at the ID22 beamline of the ESRF with incident wavelength $0.3999 \AA$. The powder was packed into a glass capillary with an outer diameter of $0.7 \mathrm{~mm}$ and spun during data acquisition with temperatures from 80 to $500 \mathrm{~K}$ controlled using an Oxford Cryostream system. High resolution neutron diffraction data were collected at the D2B beamline of the ILL with incident wavelength $1.5940 \AA$. $5 \mathrm{~g}$ of powder samples were packed into a vanadium can and diffraction patterns were collected at temperatures from 4 to $500 \mathrm{~K}$. Crystal and magnetic structures of $\mathrm{CaFe}_{3} \mathrm{O}_{5}$ were Rietveld-fitted using the FullProf Suite ${ }^{24}$. A small amount of $\mathrm{Fe}$ at the $\mathrm{Ca}$ site was found from both synchrotron $\mathrm{X}$-ray $[4.4(3) \% \mathrm{Fe}]$ and neutron $[4.0(8) \% \mathrm{Fe}]$ refinements of cation site occupancies at $500 \mathrm{~K}$. Crystal structure refinements of the two phases formed below $302 \mathrm{~K}$ in $\mathrm{CaFe}_{3} \mathrm{O}_{5}$ was possible with both the synchrotron X-ray and neutron powder diffraction data except at temperatures just below the transition ( 275 and $285 \mathrm{~K}$ ). Magnetic irreducible representation analysis was carried out using BasIrReps. Bond valence sums for each iron site were calculated using a standard method with linear interpolation to estimate mixed charge states between $\mathrm{Fe}^{2+}$ and $\mathrm{Fe}^{3+25,26}$. The crystal structure projection in Fig. la was generated using VESTA ${ }^{27}$ and the magnetic structures in Fig. 3a, b were made using FullProf Studio.

Electronic structure calculations. DFT $+U$ electronic structure calculations were performed using CASTEP $^{28}$ (v16 and 17) utilising planewaves (650 eV cutoff) and on-the-fly pseudopotentials, within the PBE approximation to exchange and correlation. Band structures (shown in Supplementary Fig. 4 and Supplementary Table 6) predict metallic charge averaged ground states for both the refined CO and CA structures at small values of the Hubbard $U$ energy, and insulating charge ordered states for both phases at large $U$. However, values in the range $2.0<$ $U<4.0 \mathrm{eV}$ simultaneously predict the charge averaged state for the CA structure and the charge ordered state for the CO structure, and hence confirm that this range is realistic for Fe oxides.

Data availability. Data that support the findings of this study have been deposited at https://doi.org/10.7488/ds/2378.

Received: 3 March 2018 Accepted: 25 June 2018

Published online: 30 July 2018

\section{References}

1. Verwey, E. J. W. Electronic conduction of magnetite $\left(\mathrm{Fe}_{3} \mathrm{O}_{4}\right)$ and its transition point at low temperatures. Nature 144, 327-328 (1939)

2. Senn, M. S., Wright, J. P. \& Attfield, J. P. Charge order and three-site distortions in the Verwey structure of magnetite. Nature 481, 173-176 (2012).

3. Dagotto, E., Hotta, T. \& Moreo, A. Colossal magnetoresistant materials: the key role of phase separation. Phys. Rep. 344, 1-153 (2001). 
4. Uehara, M., Mori, S., Chen, C. H. \& Cheong, S. W. Percolative phase separation underlies colossal magnetoresistance in mixed-valent manganites. Nature 399, 560-563 (1999).

5. Tokura, Y. Critical features of colossal magnetoresistive manganites. Rep. Prog. Phys. 69, 797-851 (2006)

6. Lavina, B. et al. Discovery of the recoverable high-pressure iron oxide $\mathrm{Fe}_{4} \mathrm{O}_{5}$. Proc. Natl Acad. Sci. USA. 108, 17281-5 (2011).

7. Ovsyannikov, S. V. et al. Charge-ordering transition in iron oxide $\mathrm{Fe}_{4} \mathrm{O}_{5}$ involving competing dimer and trimer formation. Nat. Chem. 8, 501-508 (2016).

8. Hong, K. H., McNally, G. M., Coduri, M. \& Attfield, J. P. Synthesis, crystal structure, and magnetic properties of $\mathrm{MnFe}_{3} \mathrm{O}_{5}$. Z. für Anorg. und Allg. Chem. 642, 1355-1358 (2016).

9. Hong, K. H., Arevalo-Lopez, A. M., Coduri, M., McNally, G. M. \& Attfield, J. P. Cation, magnetic, and charge ordering in $\mathrm{MnFe}_{3} \mathrm{O}_{5}$. J. Mater. Chem. C. 6, 3271-3275 (2018).

10. Evrard, O. et al. Mise en évidence de $\mathrm{CaFe}_{4} \mathrm{O}_{6}$ et détermination des structures cristallines des ferrites de calcium $\mathrm{CaFe}_{2+\mathrm{n}} \mathrm{O}_{4+\mathrm{n}}(n=1,2,3)$ : nouvel exemple d'intercroissanc. J. Solid State Chem. 35, 112-119 (1980).

11. Delacotte, C. et al. Structural transition at $360 \mathrm{~K}$ in the $\mathrm{CaFe}_{5} \mathrm{O}_{7}$ ferrite: toward a new charge ordering distribution. Inorg. Chem. 53, 10171-10177 (2014).

12. Ahn, K. H., Lookman, T. \& Bishop, A. R. Strain-induced metal-insulator phase coexistence in perovskite manganites. Nature 428, 401-404 (2004).

13. Burgy, J., Moreo, A. \& Dagotto, E. Relevance of cooperative lattice effects and stress fields in phase-separation theories for CMR manganites. Phys. Rev. Lett. 92, 3-6 (2004).

14. Guzmán-Verri, G. G., Brierley, R. T., Littlewood, P. B. Elastic interactions and control of the Mott transition. Preprint at arXiv:1701.02318 (2017).

15. Shenoy, V. B. \& Rao, C. N. R. Electronic phase separation and other novel phenomena and properties exhibited by mixed-valent rare-earth manganites and related materials. Philos. Trans. R. Soc. A 366, 63-82 (2008).

16. Attfield, J. P. Orbital molecules in electronic materials. APL Mater. 3, 1-7 (2015).

17. Woodward, P. M., Cox, D. E., Vogt, T., Rao, C. N. R. \& Cheetham, A. K. Effect of compositional fluctuations on the phase transitions in $\left(\mathrm{Nd}_{1 / 2} \mathrm{Sr}_{1 / 2}\right) \mathrm{MnO}_{3}$. Chem. Mater. 11, 3528-3538 (1999).

18. Zhu, Y. et al. Chemical ordering suppresses large-scale electronic phase separation in doped manganites. Nat. Commun. 7, 1-6 (2016).

19. Magen, C. et al. Observation of a Griffiths-like phase in the magnetocaloric compound $\mathrm{Tb}_{5} \mathrm{Si}_{2} \mathrm{Ge}_{2}$. Phys. Rev. Lett. 96, 167201 (2006).

20. Yunoki, S. et al. Phase separation in electronic models for manganites. Phys. Rev. Lett. 80, 845-848 (1998).

21. Moreo, A., Yunoki, S. \& Dagotto, E. Phase separation scenario for manganese oxides and related materials. Science 283, 2034-2040 (1999).

22. Milward, G. C., Calderon, M. J. \& Littlewood, P. B. Electronically soft phases in manganites. Nature 433, 607-610 (2005).

23. Wan, R., Jia, C. \& Zhang, W. Preparation and photoelectric properties of $\mathrm{p}$ $\mathrm{CaFe}_{2} \mathrm{O}_{4} / \mathrm{n}-\mathrm{WO}_{3}$ composites. J. Alloy Compd. 544, 1-5 (2012).

24. Rodriguez-Carvajal, J. Recent advances in magnetic structure determination by neutron powder diffraction. Physica B. 192, 55-69 (1993).

25. Attfield, J. P. Charge ordering in transition metal oxides. Solid State Sci. 8, 861-867 (2006).
26. Brown, I. D. VALENCE: a program for calculating bond valences. J. Appl. Crystallogr. 29, 479-480 (1996)

27. Momma, K. \& Izumi, F. VESTA 3 for three-dimensional visualization of crystal, volumetric and morphology data. J. Appl. Crystallogr. 44, 1272-1276 (2011).

28. Clark, S. J. et al. First principles methods using CASTEP. Z. Krist. 220, 567-570 (2005)

\section{Acknowledgements}

The authors acknowledge financial support from European Research Council (ERC), Engineering and Physical Sciences Research Council (EPSRC) and Science and Technology Facilities Council (STFC). The authors also thank Andy Fitch for help at ESRF and Gary Nichol, Graham McNally, Alex Browne and Giuditta Perversi for local support.

\section{Author contributions}

K.H.H. and J.P.A. designed the concept for this study. K.H.H., A.M.A.-L., and C.R performed the experimental work and data analysis, and J.C. performed the band structure calculations. K.H.H. and J.P.A. wrote the manuscript with inputs from all authors.

\section{Additional information}

Supplementary Information accompanies this paper at https://doi.org/10.1038/s41467 018-05363-6.

Competing interests: The authors declare no competing interests.

Reprints and permission information is available online at http://npg.nature.com/ reprintsandpermissions/

Publisher's note: Springer Nature remains neutral with regard to jurisdictional claims in published maps and institutional affiliations.

\begin{abstract}
Open Access This article is licensed under a Creative Commons Attribution 4.0 International License, which permits use, sharing, adaptation, distribution and reproduction in any medium or format, as long as you give appropriate credit to the original author(s) and the source, provide a link to the Creative Commons license, and indicate if changes were made. The images or other third party material in this article are included in the article's Creative Commons license, unless indicated otherwise in a credit line to the material. If material is not included in the article's Creative Commons license and your intended use is not permitted by statutory regulation or exceeds the permitted use, you will need to obtain permission directly from the copyright holder. To view a copy of this license, visit http://creativecommons.org/ licenses/by/4.0/.
\end{abstract}

(c) The Author(s) 2018 